\section{Case report of self-injurious behaviour (SIB) presenting as gingivitis artefacta major}

\author{
C. S. Millen ${ }^{1}$ and E. M. Roebuck ${ }^{2}$
}

VERIFIABLE CPD PAPER

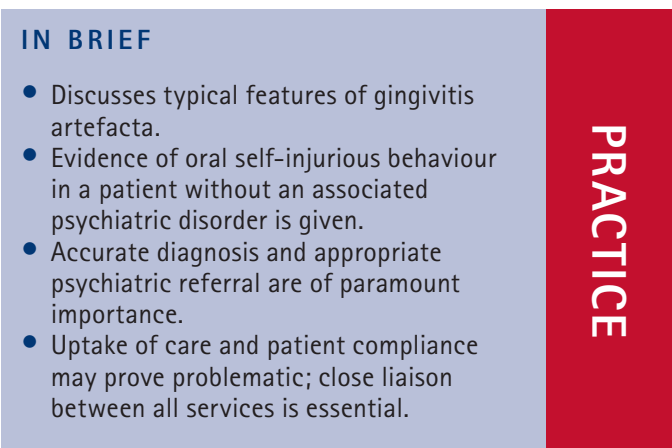

The case report described here discusses gingivitis artefacta major, an oral presentation of self-injurious behaviour, in an adolescent. On presentation, the patient knew well the ramifications of her gum scratching behaviour, however, was unable to stop. At further presentations new lesions had appeared with further bone loss. The cause of her behaviour seemed to be of psychological origin and therefore no interventive dental treatment was possible until this issue was resolved. A more preventive approach was adopted in the meantime. Referral to appropriate services from the dental profession also proved to be challenging. In conclusion, gingivitis artefacta, although rarely seen to this extent, is extremely challenging to diagnose and treat fully in a dental setting.

\section{INTRODUCTION}

Self-injurious behaviour (SIB) is described as 'self inflicted damage without suicidal intent.' ${ }^{\text {It }}$ is believed to affect $4 \%$ of adolescents in the community over a 12 month period. ${ }^{2}$ Prevalence in females aged 15-19 is greater than in males, however, this trend is reversed in the 21-24 age group. ${ }^{2}$ The relationship between SIB and genetic conditions such as Lesch-Nyhan Syndrome and autism is well documented ${ }^{3-5}$ with a prevalence of up to $40 \%$ cited where profound disabilities exist.

While the majority of the published literature in this area would appear to be case reports of individuals with a mental disability, Stewart and Kernohan ${ }^{6}$ make a differentiation between three types of injury for patients of normal intelligence:

Type A: Injuries superimposed upon a pre-existing lesion (or irritation)

Type B: Injuries secondary to another established behaviour (such as thumb sucking)

I*Clinical Lecturer/Specialist Registrar in Restorative Dentistry, ${ }^{2}$ Consultant in Paediatric Dentistry, $4^{\text {th }}$ floor Edinburgh Dental Institute, Lauriston Building, Lauriston Place, Edinburgh EH3 9YW

*Correspondence to: Dr Chris Millen

Email: dentist.millen@btinternet.com

\section{Refereed Paper}

Accepted 20 November 2008

DOI: $10.1038 /$ sj.bdj.2009.55

${ }^{\circledR}$ British Dental Journal 2009; 206: 129-131
Type C: Injuries of unknown or complex aetiology (often based upon some emotional disturbance or psychological illness).

Clinically, irrespective of any physical or mental condition, SIB has a wide range of presentations ${ }^{7}$ with $75 \%$ of injuries reported to affect the head and neck. ${ }^{8} \mathrm{~A}$ factitious dermatitis component of SIB may be found extra orally on the scalp, face or limbs. The behaviour here is more commonly reported alongside an associated psychiatric disorder and underlying emotional disturbance., ${ }^{713}$ Oral presentations include ulceration of the tongue from biting and cigarette burns, ${ }^{9}$ and scratching of the gingivae causing recession (gingivitis artefacta) with bone loss. ${ }^{3}$ In severe cases, this leads to autoextraction..$^{10,11}$

Two presentations of gingivitis artefacta, minor and major have been described. In the former, the behaviour is usually provoked by some kind of localised irritation ${ }^{12}$ and is more likely to be found in one intra-oral site only. The features of gingivitis artefacta major which facilitate the diagnosis are that the lesions are more often multiple. They do not correspond to any known disease and are mostly of a bizarre configuration with sharp outlines, surrounded by otherwise healthy tissue. In addition, the grouping and distribution of the lesions is unusual and in locations which are easily accessible by the patient. ${ }^{6}$ Clearly, in such cases, there is a need to differentiate between other possible dental diagnoses.

To date, the management of gingivitis artefacta major by the dental profession has involved the removal of the preexisting locus of irritation, prevention of a behaviour involved or appropriate psychiatric referral. ${ }^{6}$

As previously mentioned, the majority of the literature in this area refers to case reports of individuals with a mental disability. In this case report of a fifteen year old female with gingivitis artefacta major, there was no known accompanying disability or oral irritant at presentation.

\section{CASE REPORT}

A 15-year-old female attended Casualty in the local paediatric dental department with her elder sister for the management of pericoronitis affecting a partially erupted 38. Further examination revealed labial gingival recession ranging from $6-10 \mathrm{~mm}$ and associated ulceration of the reflected mucosa in the maxillary canine and mandibular incisor regions in an otherwise healthy mouth (Figs 1-3). With the exception of asthma, her medical history was clear. 
On questioning, the patient noted that she felt that she 'must get rid of the gum between the teeth' and thus she had scratched the gingivae with her nails. When her nails were short, she used any available instrument, for example, household tweezers, to achieve this.

A diagnosis of gingivitis artefacta was made and, in view of the acute symptoms from the pericoronitis, appropriate measures were introduced for the management of this, and arrangements were made for further review of the gingival condition and definitive treatment planning.

During further discussion at review, a history of parental substance abuse and neglect was given and as a result of this, the patient indicated that she had elected to move away from home to live with her sibling. Furthermore, she had had a course of Cognitive Behaviour Therapy (CBT) under the care of the Child and Adolescent Mental Health Services (CAMHS). On completion of this, she was deemed to require no further treatment. However, while gingival trauma had been inflicted, at that point in time it had not been highlighted.

At this review appointment, the following management plan was agreed: Referral to CAMHS for further assessment and management, and assessment of the available options for managing the gingival and periodontal tissues.

There then followed a six-month period of failed appointments. When contact was re-established, the previously diagnosed lesions had increased in size and new lesions in the 33 and 43 regions were evident (Figs 4-5). There was clear radiographic evidence of interdental and labial bone loss in these areas. All involved teeth gave positive responses to sensibility testing.

A new medical history revealed additional information: an allergy to animal fur, slight narcolepsy and occasional dehydration leading to severe headaches and nausea and which had necessitated hospitalisation on one occasion within the six month intervening period. The patient also indicated that she had had no contact from CAMHS and was planning to relocate to her home city. She indicated that she was happy for a referral to the local specialist services to be made.
The increasing damage to the toothsupporting structures was of great concern. The management options were reviewed and an interventive measure such as free-gingival graft surgery was felt to be contra-indicated at that time. A preventive approach was adopted and impressions were taken for the fabrication of gingival shields. However, the appointment for delivery of these was missed and several attempts to contact the patient were unsuccessful.

On subsequent discussion with CAMHS, it became apparent that the offer of an appointment through their patient-focussed booking scheme had not been taken up and thus the patient had been discharged from their care. However, all related correspondence had been directed to her general medical practitioner and not the referring specialist paediatric dental practitioner. During further discussion, it transpired that this had arisen because of the automated nature of the booking system and the general medical practitioner was the usual source of such referrals.

Appropriate referrals to services in the area of her new residence have now been made. Contact with these services has since been made and the patient has now accessed care. However, similar problems in attendance as described in this report, have been experienced.

\section{DISCUSSION}

Despite experiencing pain, acknowledging the SIB and expressing a desire for help to overcome her difficulties, the offer of care within the dental and CAMHS services was not taken up. The reasons for this can only be speculated upon. However, previous studies looking at the profile of patients who miss appointments have highlighted this age group (17-40 years) and a history of psychological problems as factors. ${ }^{14}$ In this case a psychiatric referral was made, but as demonstrated here and elsewhere in the literature, breakdown of these referrals and non-attendance in this patient group can be high. ${ }^{15}$

In addition, details of the history were not immediately revealed, thus highlighting the difficulties there can be obtaining a comprehensive history in such cases. This may help to explain

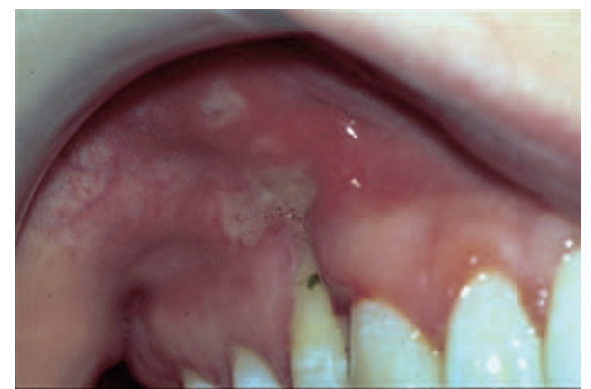

Fig. 1 Maxillary right quadrant; gingival recession

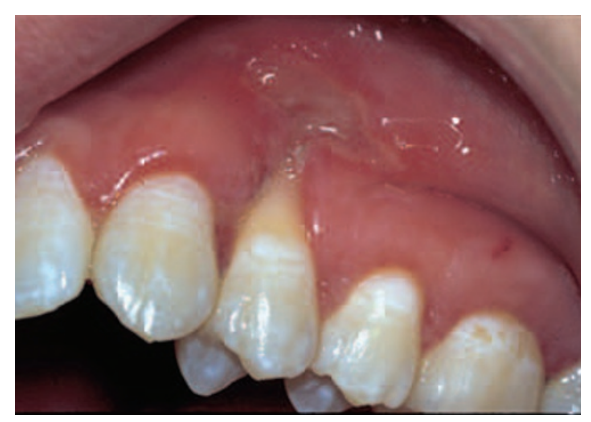

Fig. 2 Maxillary left quadrant; gingival recession

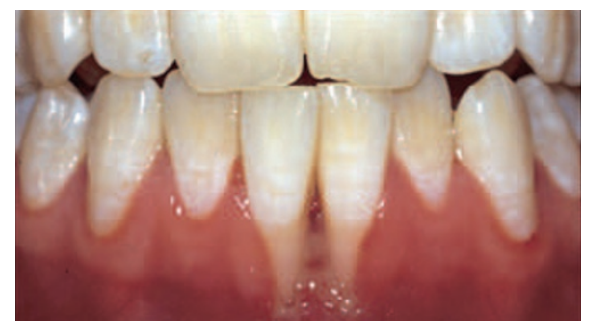

Fig. 3 Mandibular incisor region; gingival recession



Fig. 4 Further recession and bone loss; mandibular left quadrant

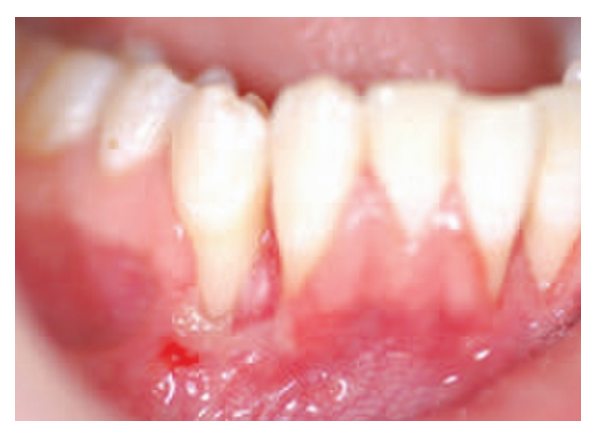

Fig. 5 Further recession and bone loss; mandibular right quadrant 
that, while under hospital medical care for dehydration, the patient's SIB failed to be noticed.

Contemporary dentistry compels us to approach our patients holistically. While we are becoming more accustomed to the idea of providing smoking cessation advice and asking about our patients' social circumstances, assessing them psychologically is a difficult, and inappropriate, path for the dentist to tread. Therefore an avenue for referrals to appropriate services such as those mentioned here is essential. However, because of the mechanism of dealing with patient focussed bookings within CAHMS, there was a breakdown in communication between it and the referrer, despite the fact that the referring Specialist Paediatric Dental Centre was a frequent user of the service for other conditions such as the management of severe dental anxiety. This void in communication is hopefully rare; however, it highlights the need for additional checks to be put in place to ensure that all clinicians involved in a patient's care are kept fully informed of progress.

In this case, in an attempt to limit further destruction of the tissues, two designs of removable gingival shield were constructed. While their removable nature necessitated a degree of compliance, it was not possible to determine how effective they would have been because of the failed appointments. It was hoped that with the patient's request for help and her referral to CAHMS, the use of the shields would act as an adjunct to any input from CAHMS. However, at this point in time, the efficacy of the proposed treatment cannot be speculated on.

At the patient's most recent examination, gingival recession of 6-11 $\mathrm{mm}$ with associated bone loss of the six affected teeth was evident. If this continues, one can only assume that the involved teeth will ultimately be lost and other areas may be similarly damaged.

This case highlights both the desperate need for accurate diagnosis on behalf of the dental profession, and the management difficulties encountered.

Full written valid consent was obtained for the publication of this report and its accompanying photographs.

\section{What this case report adds}

- Demonstration of the difficulties in treating gingivitis artefacta major

- Evidence of oral SIB in a patient without an associated psychiatric disorder but with a subtle emotional disturbance

- Uptake of care and patient compliance may prove problematic and therefore close liaison between all services is essential.

\section{Why this case report is important to dentists}

- It illustrates the typical features of gingivitis artefacta major

- When a diagnosis of gingivitis artefact major is made, the presence of an underlying emotional or psychiatric issue must be considered

- Accurate diagnosis and appropriate psychiatric referral are of paramount importance.

1. Vogel L D. When children put their fingers in their mouths. Should parents and dentists care? NY State Dent J 1998; 64: 48-53.

2. Scottish Development Centre for Mental Health \& Research Unit in Health, Behaviour and Change, University of Edinburgh. WRITTEN ON THE BODY, A review of literature on self-cutting. National Inquiry into Self Harm among Young People, Executive Summary, June 2005.

3. Medina A C, Sogbe R, Gomez-Ray A M, Mata $M$. Factitial oral lesions in an autistic paediatric patient. Int J Paediatr Dent 2003; 13: 130.

4. Cusumano FJ, Penna K, Panossian G. Prevention of oral self-mutilation in patients with LeschNyhan syndrome: review of literature. J Dent Child 2001; 68: 175-178.

5. Chien $L R$, Liu J. Successful treatment of selfinflicted oral mutilation using an acrylic splint retained by head gear. Pediatr Dent 1996 18: 408-410.

6. Stewart D J, Kernohan D C. Self-inflicted gingival injuries gingivitis artefacta, factitial gingivitis Dent Practitioner 1972; 22: 418-426.

7. Welbury R. Paediatric dentistry. Oxford University Press, 2003.

8. Van Moffaert M. Localization of self-inflicted dermatological lesions: what do they tell the dermatologist? Acta Derm Venereol 1991; 156: 23-27.

9. Blanton $\mathrm{PL}$, Hurt W C, Largent M D. Oral factitious injuries. J Periodontol 1977; 48: 33-37.

10. Leksell E, Edvardson S. A case of Tourette syndrome presenting with oral self-injurious behaviour. Int J Paediatr Dent 2005; 15: 370-374.

11. Plesset D D. Autoextraction. Oral Surg 1959; 12: 302-305.

12. Spencer R J, Haria S, Evans R D. Gingivitis artefacta - a case report of a patient undergoing orthodontic treatment. Br J Orthod 1999; 26: 93-96.

13. Golden S, Chosack A. Oral manifestations of a psychological problem. J Periodontol 1964 35: 349.

14. George A, Rubin G. Non-attendance in general practice: a systematic review and its implications for access to primary health care. Fam Pract 2003; 20: 178-184.

15. McKay M M, Stoewe J, McCadam K, Gonzales J. Increasing access to child mental health services for urban children and their caregivers. Health Soc Work 1998; 23: 9-15. 